\title{
Nephrolithiasis and risk of hypertension: a meta-analysis of observational studies
}

\author{
Weifeng Shang ${ }^{1}$, Yuanyuan $\mathrm{Li}^{2}$, Yali Ren ${ }^{3}, \mathrm{Yi}_{\mathrm{Yang}}{ }^{4}$, Hua Li ${ }^{1}$ and Junwu Dong ${ }^{1 *}$
}

\begin{abstract}
Background: Observational studies have demonstrated an association between nephrolithiasis and hypertension. The aim of this meta-analysis was to summarize all available evidence.

Methods: PubMed, EMBASE, the Cochrane Central Register of Controlled Trials databases, and the reference lists of relevant articles were searched to identify observational studies that reported study-specific risk estimates comparing the risk of hypertension in patients with nephrolithiasis. We used a random-effect model to pool the study-specific risk estimates. We also assessed the potential heterogeneity by subgroup analyses, meta-regression analyses, and sensitivity analyses.
\end{abstract}

Results: A total of 7 articles including 9 studies ( $n=313,222$ participants) were eventually identified in this meta-analysis. In comparison with the patients who did not have nephrolithiasis, nephrolithiasis significantly increased the risk of hypertension (OR, 1.43; 95\% Cl, 1.30-1.56), with significant heterogeneity between these studies $\left(P^{2}=83.5 \%, P<0.001\right)$. The heterogeneity reduced in subgroups of cohort studies, USA, large sample size trials, men, and adjustment for confounding factors $\geq 5$. Sensitivity analysis further demonstrated the results to be robust.

Conclusions: Nephrolithiasis is associated with increased risk of hypertension. Future randomized, high-quality clinical trials are encouraged to definitively clarify the relationship between nephrolithiasis and hypertension, which may influence clinical management and primary prevention of hypertension in nephrolithiasis patients.

Keywords: Kidney stone, Nephrolithiasis, Hypertension, Meta-analysis

\section{Background}

Nephrolithiasis is a common condition, with the prevalence varying by age and sex. The disease usually presents in men aged 60 to 69 , with a prevalence rate of approximately 1.7 to $8.8 \%$ worldwide [1, 2]. Hypertension is defined as persistent elevation of systematic arterial blood pressure (systolic pressure $\geq 140 \mathrm{mmHg}$ and/or diastolic pressure $\geq 90 \mathrm{mmHg}$ ). It is an extremely common cardiovascular disease, affecting over $30 \%$ of young adults and $70 \%$ of elderly individuals [3]. Hypertension is also a silent yet dangerous disease. Despite intensive studies aimed at identifying risk factors for hypertension, the exact pathogenic mechanisms of hypertension often are unclear. Nevertheless, there is growing evidence supporting that nephrolithiasis largely

\footnotetext{
* Correspondence: junwudongwuhan@163.com

${ }^{1}$ Department of Nephrology and Rheumatology, The Forth Hospital of Wuhan Affiliated with Tongji Medical College, Huazhong University of Science and Technology, Wuhan, Hubei 430030, People's Republic of China Full list of author information is available at the end of the article
}

contribute to the occurrence of hypertension. Since the association between arterial hypertension and nephrolithiasis was described in 1965 for the first time by Tibblin [4], much effort has been devoted to this field. Data from several observational studies suggested a risk of hypertension in nephrolithiasis patients of 1.24-1.96 compared to the general population [5-11]. A previous review performed by Cupisti et al. [12] has shown the current understanding of the potential link between nephrolithiasis and the occurrence of hypertension, but no meta-analysis has been used to examine the relationship.

Given the fact that individual studies may have insufficient statistical power because of sample size, we performed a meta-analysis to collect all beneficial evidence to assess the risk of hypertension among nephrolithiasis patients, which may emphasize the importance of considering additional intervention methods in this area. 


\section{Methods}

Our study was conducted in accordance with the Preferred Reporting Items for Systematic Reviews and Meta-Analyses (PRISMA) statement checklist [13].

\section{Search strategy}

PubMed, EMBASE, and Cochrane Library databases were searched for observational studies to March 18, 2017. The used search terms were as follows: "renal stones" or "renal stone" or "kidney stones" or "kidney stone" or "nephrolithiasis" or "calculi" and "hypertension" or "blood pressure" and "risk" or "incidence" or "epidemiology". Furthermore, we searched reference lists of all included studies for additional eligible studies. Two of the authors (WS and YL) independently screened titles and abstracts, analyzed full-text articles, and ascertained the final eligible records. Conflicting results were resolved by discussion. We merged retrieved citations using EndNote X7.

\section{Inclusion and exclusion criteria}

The inclusion criteria were: (1) the study design was a cross-sectional, case-control, or cohort study; (2) identified nephrolithiasis as exposure, including medical records, questionnaire, direct interview etc.; (3) the outcome measure was hypertension, including medical records, questionnaire, blood pressure measurement, direct interview etc.; and (4) odds ratio (OR) or hazard ratio (HR) or risk ratio (RR), and the corresponding 95\% confidence interval (CI) were reported or could be calculated. Reviews, letters, case reports, and animal studies were excluded.

\section{Data extraction}

Study characteristics were extracted by two authors (WS and YL) separately as follows: first author's name, publication year, country origin, study design, sample, average age, proportion of men, method of nephrolithiasis and hypertension diagnosis, and adjustment factors. When needed, we contacted the original author for clarification.

\section{Quality assessment}

We evaluated the quality of studies using the Strengthening the Reporting of Observational Studies in Epidemiology (STROBE) statement [14]. Two authors (WS and YR) performed the quality assessment independently and disagreements were resolved by discussion.

\section{Statistical analyses}

The study-specific most adjusted HR, RR or OR was used to compute a summary OR and its 95\%CI. HRs and RRs were directly considered as ORs [15]. Heterogeneity of ORs among studies was assessed using the
Chi-squared based on Q-statistic test $(P<0.10)$ and quantified by $\mathrm{I}^{2}$ statistic. $\mathrm{I}^{2}$ values were considered to represent insignificant (0-25\%), low (26-50\%), moderate $(51-75 \%)$, and high $(>75 \%)$ heterogeneity [16]. The random-effects model was used to calculate the combined risk estimates. Subgroup analysis and univariable random effects meta-regression were further conducted to explore the potential source of heterogeneity. Stratified analyses were conducted based on study design (cohort or cross-sectional), region (USA or non-USA), sample size $(<35,000$ or $\geq 35,000)$, gender (men or women), and the number of confounders adjusted for $(<5$ or $\geq 5)$. We conducted sensitivity analyses to assess the influence of a study on the pooled effect estimate by recalculating the pooled OR with removal of one study in each turn. Reporting bias was evaluated using Egger's test [17]. All meta-analyses were performed by the STATA (version 10.0, Stata Corporation, College Station, TX, USA). $P<0.05$ in 2 -tailed test was considered to be statistically significant.

\section{Results}

Study selection, characteristics, and quality

As shown in Fig. 1, our literature search returned 2327 results for relevant articles, and the full text retrieved for 85 articles. Finally, we identified 9 observational studies, based on 7 articles.

The main characteristic of the studies included are presented in Table 1. Included studies were published during 1998-2017. These articles included 4 cohort studies, and 5 cross-sectional studies. Of these studies, six were conducted in United States, one in Italy, one in Portugal, and one in Japan. The primary analysis included data for 313,222 participants derived from 9 observational studies that reported an association between nephrolithiasis and the risk of hypertension.

According to the STROBE, all but one included studies were of high quality (Additional file 1: Table S1).

\section{Nephrolithiasis and risk of hypertension}

As shown in Fig. 2, the multivariate-adjusted OR of hypertension within the 9 individual study populations ranged between 1.24 and 1.96, with an overall multivariateadjusted OR of 1.43 (95\% CI, 1.30-1.56). Significant heterogeneity was observed $\left(I^{2}=83.5 \%, P<0.001\right)$.

\section{Subgroup analyses}

In most cases, low-to-high heterogeneity was still present in stratified analyses unless adjustment for confounding factors was more than $5\left(I^{2}=0 \%\right)$. We used meta-regression to explore the sources of heterogeneity and found that study design, resign, sample size, gender, and adjustment for confounding factors may be potential sources of heterogeneity (Table 2). 


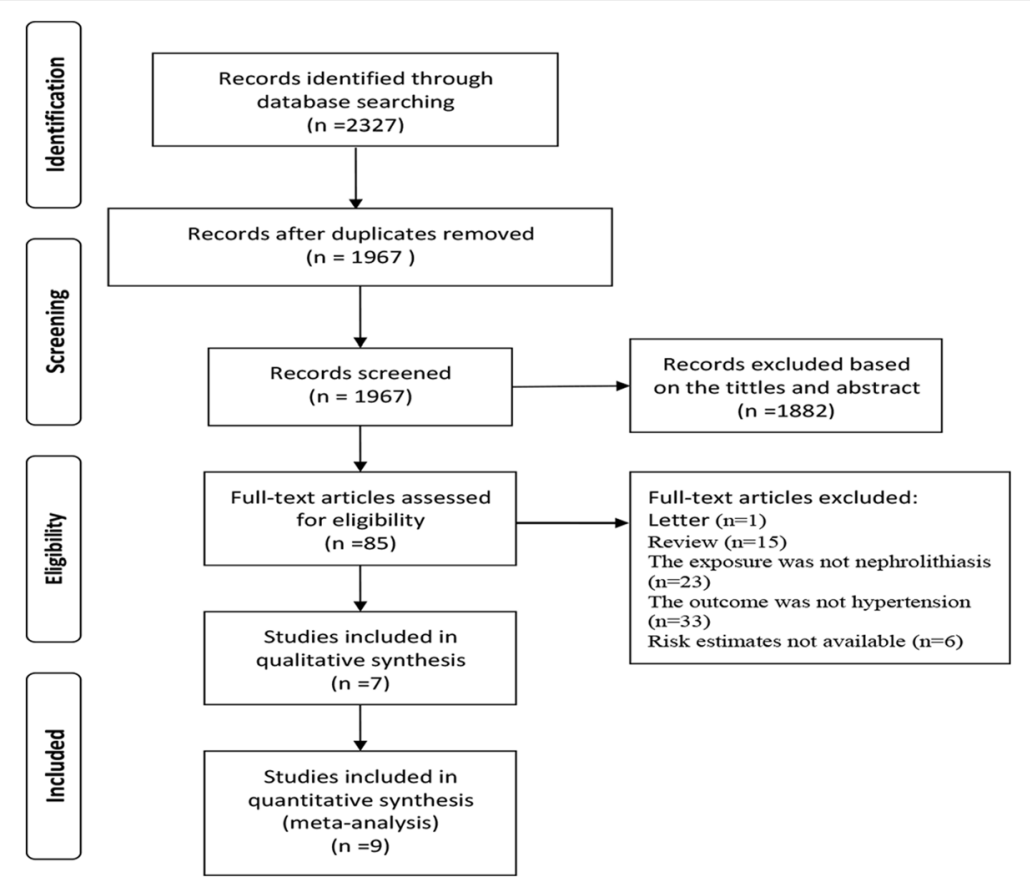

Fig. 1 Flow chart of study selection

\section{Sensitivity analyses and reporting bias}

Sensitivity analyses were performed by excluding one study at a time. And they indicated that the omission of any of the studies led to changes in estimates between 1.34 (95\% CI: $1.27-1.40$ ) and 1.47 (95\% CI: 1.32-1.64). The changes were not significant. However, deletion of the Domingos et al.'s study reduced the heterogeneity from high to low levels (Additional file 2: Table S2). The $P$ value of Egger's test was 0.134 , suggesting that there was no publication bias statistically.

\section{Discussion}

To the best of our knowledge, this study is the first meta-analysis to present hypertension risk in patients with a history of nephrolithiasis. We confirmed nephrolithiasis was associated with an increased risk of hypertension. The risk of hypertension remained pronounced in all subgroups.

As with other published meta-analyses of this type $[18,19]$, our study has a high level of heterogeneity. We constructed subgroup analyses and metaregression analyses to explore sources of heterogeneity. In subgroup analyses, the heterogeneity reduced in patients within cohort studies, USA, large sample size trials, men, and adjustment for confounding factors $\geq 5$. This conclusion is supported by the results of the meta-regression, which showed that study design, resign, sample size, gender, and adjustment for confounding factors may be potential sources of heterogeneity. In addition, different follow-up time and adjust factors may be also the source of heterogeneity.

Note that female patients with kidney stones showed much higher risk for hypertension than male patients in our study. The underlying pathophysiology remains unclear. However, the differences by sex are not infrequent. A similar finding has also been observed in a metaanalysis on the association of nephrolithiasis and risk of incident chronic kidney disease (CKD) [18]. Similarly, a review also demonstrated a statistically significant increased risk of coronary heart disease in female patients with prior nephrolithiasis, but there was no significant association in male patients with prior nephrolithiasis [20]. Of note, Madore et al.'s study indicated that both women and men with hypertension at baseline were not more likely to develop nephrolithiasis during the follow-up $[5,6]$. Due to the limited data, further studies are needed to direct the sex difference in hypertension response to nephrolithiasis.

The relation between nephrolithiasis and hypertension is rather unclear, but after our complete literature retrieval, we found several potential reasons which may explain the observed associations. First, alterations in calcium metabolism maybe have an important role in the pathogenesis of both nephrolithiasis and hypertension [21, 22]. Second, the traits of metabolic syndrome are factors highly prevalent in hypertensives as well as in kidney stone formers, so insulin resistance may be a common pathophysiological mechanism [23, 24]. Third, CKD is a condition which may occur more frequently in 


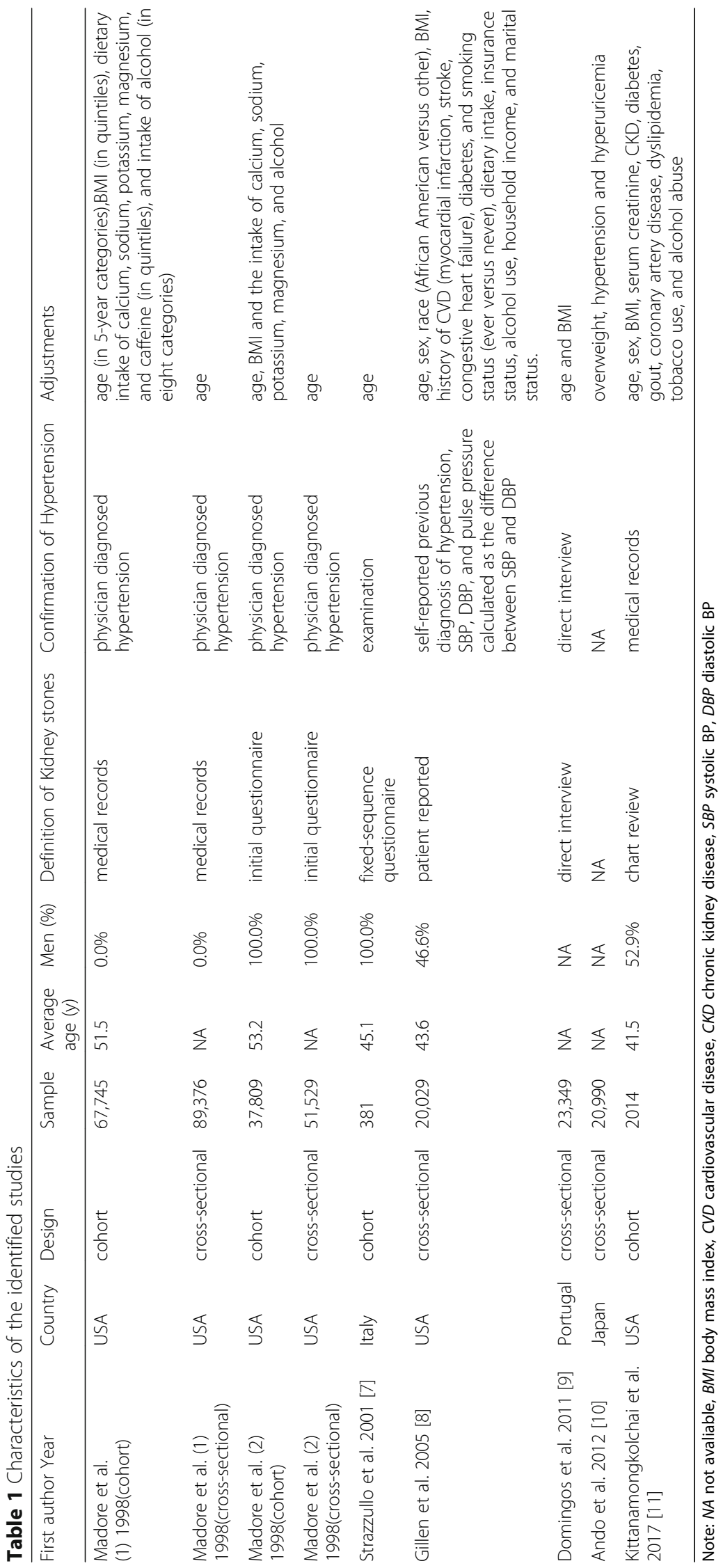




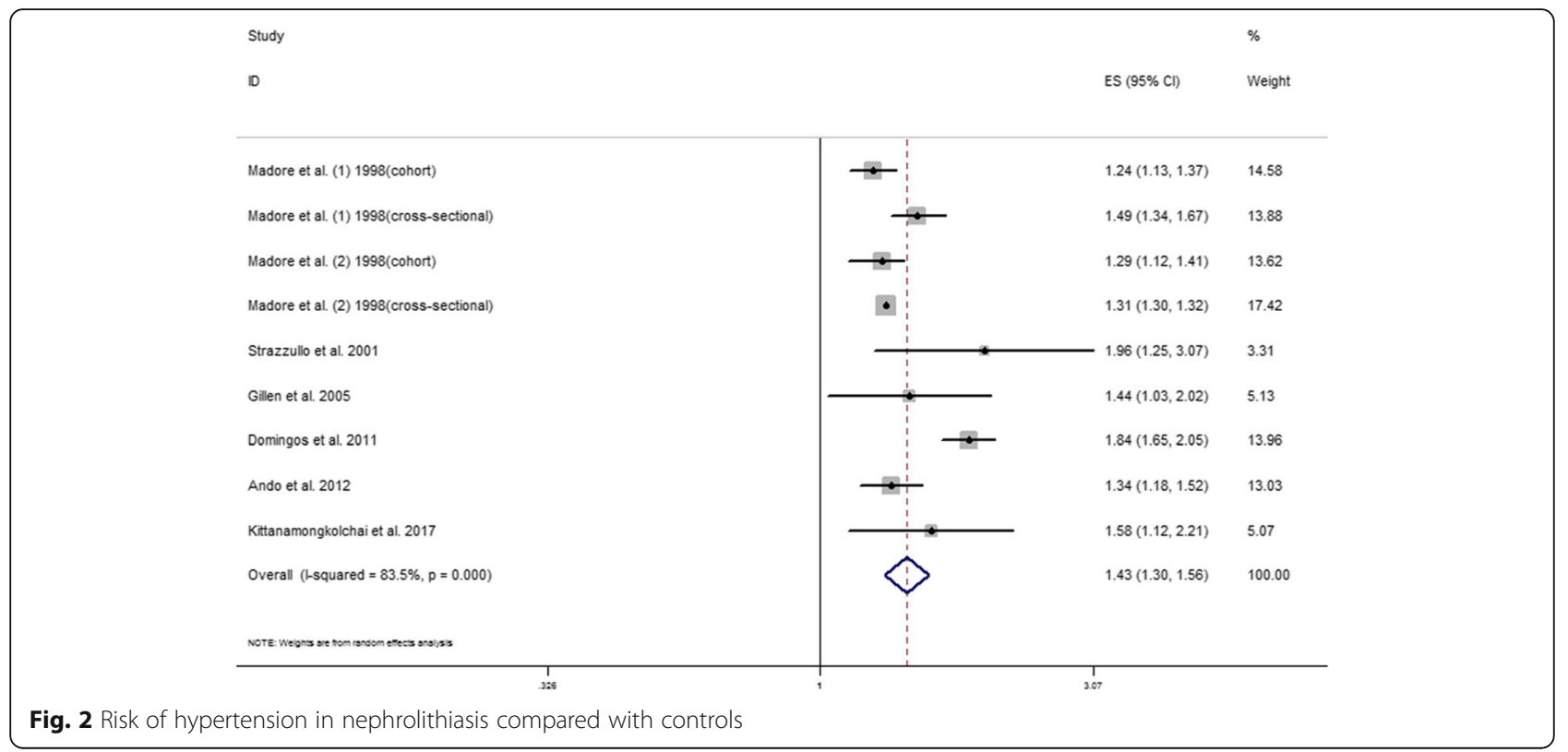

nephrolithiasis patients and in hypertensive patients. Therefore, CKD may be another factor involved in the linkage between nephrolithiasis and hypertension. Finally, inflammation and oxidative stress have been recently hypothesized as possible links between stone disease and hypertension [25]. Obviously, all of these potential reasons are comorbidities in nephrolithiasis and hypertension. However, more medical research is needed to explore and test the relevant presumption.

Table 2 Subgroup analyses of hypertension in patients with kidney stones

\begin{tabular}{|c|c|c|c|c|c|}
\hline Subgroup & No. of studies & OR $(95 \% \mathrm{Cl})$ & $1^{2}(\%)$ & $P^{a}$ & $P^{\mathrm{b}}$ \\
\hline \multicolumn{6}{|l|}{ Study design } \\
\hline Cohort & 4 & $1.33(1.18,1.49)$ & 43.8 & 0.148 & \multirow[t]{2}{*}{0.518} \\
\hline Cross-sectional & 5 & $1.47(1.28,1.70)$ & 90.7 & $<0.001$ & \\
\hline \multicolumn{6}{|l|}{ Region } \\
\hline USA & 5 & $1.33(1.26,1.40)$ & 37.8 & 0.154 & \multirow[t]{2}{*}{0.106} \\
\hline Non-USA & 3 & $1.64(1.26,2.14)$ & 86.3 & 0.001 & \\
\hline \multicolumn{6}{|l|}{ Sample size } \\
\hline$<35,000$ & 5 & $1.59(1.32,1.92)$ & 73.4 & 0.005 & \multirow[t]{2}{*}{0.079} \\
\hline$\geq 35,000$ & 4 & $1.32(1.25,1.40)$ & 54.3 & 0.087 & \\
\hline \multicolumn{6}{|l|}{ Gender } \\
\hline Men & 4 & $1.31(1.25,1.37)$ & 13.4 & 0.326 & \multirow[t]{2}{*}{0.547} \\
\hline Women & 3 & $1.43(1.21,1.69)$ & 78.2 & 0.010 & \\
\hline \multicolumn{6}{|c|}{ Adjustment for confounding factors } \\
\hline$<5$ & 5 & $1.51(1.30,1.76)$ & 91.2 & $<0.001$ & \multirow[t]{2}{*}{0.275} \\
\hline$\geq 5$ & 4 & $1.32(1.14,1.52)$ & 0 & 0.497 & \\
\hline
\end{tabular}

$O R$ odds ratio, $\mathrm{Cl}$ confidence interval

${ }^{a} P$ value for heterogeneity among studies assessed with Cochran's $Q$ test

${ }^{\mathrm{b}} P$ value for interaction evaluated by meta-regression models
Several limitations of this meta-analysis should be pointed out. First, significant heterogeneity was detected in the nephrolithiasis and hypertension, the differences in characteristics of populations, study designs, sample size, men (\%), diagnostic criteria, and adjusted confounders may contribute to the high heterogeneity. For example, the diagnosis of hypertension was inferred from self-reported blood pressure or patient questionnaire in two cross-sectional studies (Gillen et al. [8] and Domingos et al. [9]), which may bias the true incidence of hypertension. Second, we had no access to the information on the total number or type of nephrolithiasis. Therefore, we could not evaluate the association between different types of nephrolithiasis and hypertension. Third, most of the studies included were partially representatives of western countries, and thus extrapolating results to other parts of the world should be interpreted cautiously. Fourth, no publication bias was detected statistically in our study, but potential publication bias could not be completely ignored, given the fact that studies with null results tend not to be published. Finally, although all the included studies controlled for several known risk factors for hypertension, residual confounding cannot be excluded because the results of our study were based on observational studies.

\section{Conclusions}

Our study demonstrates that nephrolithiasis is significantly associated with increased risk of hypertension. Well-designed randomized controlled trials are necessary to elucidate the underlying mechanism and will provide more effective preventive and therapeutic measures. Our study has important implications for public health, which 
emphasizes that clinicians pay attention to the potential association between nephrolithiasis and hypertension.

\section{Additional files}

Additional file 1: Table S1. Strengthening the Reporting of Observational Studies in Epidemiology (STROBE) statement scores of the included published studies. (DOCX $17 \mathrm{~kb}$ )

Additional file 2: Table S2. Sensitivity analysis. (DOC $28 \mathrm{~kb}$ )

\section{Abbreviations}

Cl: 95\% confidence; HR: Hazard ratio; NOS: Newcastle-ottawa scale; OR: Odds ratio; PRISMA: Preferred reporting items for systematic reviews and metaanalyses; RR: Risk ratio; STROBE: Strengthening the Reporting of Observational Studies in Epidemiology

\section{Acknowledgements}

Not applicable.

\section{Funding}

No funding was obtained for this study.

\section{Availability of data and materials}

All data that support the conclusions of this manuscript are included within the article.

\section{Authors' contributions}

DJW conceived and designed the study. SWF, RYL, and LYY screened the abstract and full text, extracted data, assessed studies and drafted the manuscript. SWF, LYY, YY, and RYL performed statistical analyses. LH, and DJW revised the manuscript. All authors read the manuscript and approved the final version

\section{Ethics approval and consent to participate}

Not applicable.

\section{Consent for publication}

Not applicable.

\section{Competing interests}

The authors declare that they have no competing interests.

\section{Publisher's Note}

Springer Nature remains neutral with regard to jurisdictional claims in published maps and institutional affiliations.

\section{Author details}

'Department of Nephrology and Rheumatology, The Forth Hospital of Wuhan Affiliated with Tongji Medical College, Huazhong University of Science and Technology, Wuhan, Hubei 430030, People's Republic of China.

${ }^{2}$ Department of Respiratory Medicine, The Forth Hospital of Wuhan Affiliated with Tongji Medical College, Huazhong University of Science and Technology, Wuhan, Hubei 430030, People's Republic of China. ${ }^{3}$ Department of Medical Affaires, Liyuan Hospital Affiliated to Tongji Medical College, Huazhong University of Science and Technology, Wuhan, Hubei 430030, People's Republic of China. ${ }^{4}$ Department of Nephrology, Tongji Hospital Affiliated to Tongji Medical College, Huazhong University of Science and Technology, Wuhan, Hubei, China.

Received: 24 June 2017 Accepted: 17 November 2017 Published online: 29 November 2017

\section{References}

1. Lieske JC, Pena de la Vega LS, Slezak JM, Bergstralh EJ, Leibson CL, Ho KL, Gettman MT. Renal stone epidemiology in Rochester, Minnesota: an update. Kidney Int. 2006;69(4):760-4.

2. Scales CD Jr, Smith AC, Hanley JM, Saigal CS. Prevalence of kidney stones in the United States. Eur Urol. 2012;62(1):160-5.
3. Abel N, Contino K, Jain N, Grewal N, Grand E, Hagans I, Hunter K, Roy S. Eighth joint National Committee (JNC-8) guidelines and the outpatient Management of Hypertension in the African-American population. N Am J Med Sci. 2015;7(10):438-45.

4. Tibblin G. A population study of 50-year-old men. An analysis of the nonparticipation group. Acta Med Scand. 1965;178(4):453-9.

5. Madore F, Stampfer MJ, Willett WC, Speizer FE, Curhan GC. Nephrolithiasis and risk of hypertension in women. Am J Kidney Dis. 1998;32(5):802-7.

6. Madore F, Stampfer MJ, Rimm EB, Curhan GC. Nephrolithiasis and risk of hypertension. Am J Hypertens. 1998;11(1 Pt 1):46-53.

7. Strazzullo P, Barba G, Vuotto P, Farinaro E, Siani A, Nunziata V, Galletti F, Mancini M, Cappuccio FP. Past history of nephrolithiasis and incidence of hypertension in men: a reappraisal based on the results of the Olivetti prospective heart study. Nephrol Dial Transplant. 2001;16(11):2232-5.

8. Gillen DL, Coe FL, Worcester EM. Nephrolithiasis and increased blood pressure among females with high body mass index. Am J Kidney Dis. 2005;46(2):263-9.

9. Domingos F, Serra A. Nephrolithiasis is associated with an increased prevalence of cardiovascular disease. Nephrol Dial Transplant. 2011;26:864-8.

10. Ando R, Nagaya T, Suzuki S, Okada A, Yasui T, Tozawa K, Takahashi H, Kawai M, Kohri K. Positive associations of current and past history of kidney stones with overweight, hypertension, hyperuricemia and chronic kidney disease in a screened population. Urology. 2012;1:S51.

11. Kittanamongkolchai W, Mara KC, Mehta RA, Vaughan LE, Denic A, Knoedler JJ, Enders FT, Lieske JC, Rule AD. Risk of hypertension among first-time symptomatic kidney stone formers. Clin J Am Soc Nephrol. 2017;12(3):476-82

12. Cupisti A, D'Alessandro C, Samoni S, Meola M, Egidi MF. Nephrolithiasis and hypertension: possible links and clinical implications. J Nephrol. 2014;27(5):477-82

13. Moher D, Liberati A, Tetzlaff J, Altman DG. Preferred reporting items for systematic reviews and meta-analyses: the PRISMA statement. PLoS Med. 2009;6(7):e1000097.

14. von Elm E, Altman DG, Egger M, Pocock SJ, Gotzsche PC, Vandenbroucke $J P$. The strengthening the reporting of observational studies in epidemiology (STROBE) statement: guidelines for reporting observational studies. Lancet (London, England). 2007;370(9596):1453-7.

15. Greenland S. Quantitative methods in the review of epidemiologic literature. Epidemiol Rev. 1987;9:1-30.

16. Higgins JP, Thompson SG, Deeks JJ, Altman DG. Measuring inconsistency in meta-analyses. BMJ (Clin Research ed). 2003;327(7414):557-60.

17. Egger M, Davey Smith G, Schneider M, Minder C. Bias in meta-analysis detected by a simple, graphical test. BMJ (Clinical Research ed). 1997; 315(7109):629-34

18. Shang W, Li L, Ren Y, Ge Q, Ku M, Ge S, Xu G. History of kidney stones and risk of chronic kidney disease: a meta-analysis. PeerJ. 2017;5:e2907.

19. Shi HB, Tang B, Liu YW, Wang XF, Chen GJ. Alzheimer disease and cancer risk: a meta-analysis. J Cancer Res Clin Oncol. 2015;141(3):485-94.

20. Ferraro PM, Taylor EN, Eisner BH, Gambaro G, Rimm EB, Mukamal KJ, Curhan GC. History of kidney stones and the risk of coronary heart disease. JAMA. 2013:310(4):408-15.

21. Strazzullo P, Nunziata V, Cirillo M, Giannattasio R, Ferrara LA, Mattioli PL, Mancini M. Abnormalities of calcium metabolism in essential hypertension. Clin Sci (London, England: 1979). 1983;65(2):137-41.

22. Strazzullo P, Mancini M. Hypertension, calcium metabolism, and nephrolithiasis. Am J Med Sci. 1994:307(Suppl 1):S102-6.

23. Obligado SH, Goldfarb DS. The association of nephrolithiasis with hypertension and obesity: a review. Am J Hypertens. 2008;21(3):257-64.

24. Kohjimoto Y, Sasaki Y, Iguchi M, Matsumura N, Inagaki T, Hara I. Association of metabolic syndrome traits and severity of kidney stones: results from a nationwide survey on urolithiasis in Japan. Am J Kidney Dis. 2013:61(6):923-9.

25. Khan SR. Is oxidative stress, a link between nephrolithiasis and obesity, hypertension, diabetes, chronic kidney disease, metabolic syndrome? Urol Res. 2012:40(2):95-112. 